\title{
Time to appropriate antibiotic therapy is a predictor of outcome in patients with bloodstream infection caused by KPC- producing Klebsiella pneumoniae
}

Marco Falcone ${ }^{1 *}$, Matteo Bassetti ${ }^{2}$, Giusy Tiseo ${ }^{1}$, Cesira Giordano ${ }^{3}$, Elia Nencini ${ }^{4}$, Alessandro Russo $^{1}$, Elena Graziano ${ }^{5}$, Enrico Tagliaferri ${ }^{1}$, Alessandro Leonildi ${ }^{3}$, Simona Barnini ${ }^{3}$, Alessio Farcomeni ${ }^{6}$ and Francesco Menichetti ${ }^{1}$

\begin{abstract}
Background: Bloodstream infections (BSIs) by Klebsiella pneumoniae carbapenemase (KPC)-producing Klebsiella pneumoniae (Kp) are associated with high mortality. The aim of this study is to assess the relationship between time to administration of appropriate antibiotic therapy and the outcome of patients with BSI due to KPC-Kp hospitalized in intensive care unit (ICU).

Methods: An observational study was conducted in the ICUs of two academic centers in Italy. Patients with KPC-Kp bacteremia hospitalized between January 2015 to December 2018 were included. The primary outcome was the relationship between time from blood cultures $(\mathrm{BC})$ collection to appropriate antibiotic therapy and 30-day mortality. The secondary outcome was to evaluate the association of different treatment regimens with 30-day mortality and a composite endpoint (30-day mortality or nephrotoxicity). A Cox regression analysis to identify factors independently associated with 30-day mortality was performed. Hazard ratio (HR) and 95\% confidence interval (Cl) were calculated.

Results: A total of 102 patients with KPC-Kp BSI were included. The most common sources of infection were intraabdominal (23.5\%), urinary tract (20.6\%), and skin and skin structure (17.6\%). The 30-day mortality was $45 \%$. Median time to appropriate antibiotic therapy was shorter in patients who survived (8.5 h [IQR 1-36]) versus those who died (48 $\mathrm{h}[\mathrm{IQR} 5-108], p=0.014)$. A propensity score matching showed that receipt of an in vitro active therapy within $24 \mathrm{~h}$ from $B C$ collection was associated with lower 30-day mortality ( $H R=0.36,95 \% \mathrm{Cl}: 0.188-0.690, p=0.0021)$. At Cox regression analysis, factors associated with 30-day mortality were primary bacteremia (HR 2.662 [95\% Cl 1.118-6.336], $p=0.027)$, cardiovascular disease (HR 2.196 [95\% Cl 1.082-4.457], $p=0.029)$, time (24-h increments) from BC collection to appropriate therapy (HR $1.382[95 \% \mathrm{Cl} 1.132-1.687], p=0.001$ ), SOFA score (HR 1.122 [95\% Cl 1.036-1.216], $p=0.005$ ), and age (HR 1.030 [95\% Cl 1.006-1.054], $p=0.012$ ). Ceftazidime-avibactam-containing regimens were associated with reduced risk of composite endpoint (30-day mortality OR nephrotoxicity) (HR 0.231 [95\% Cl 0.071-0.745], $p=0.014$ ) compared to colistin-containing regimens.
\end{abstract}

Conclusions: Time to appropriate antibiotic therapy is an independent predictor of 30-day mortality in patients with KPC-Kp BSI. Appropriate antibiotic therapy should begin within $24 \mathrm{~h}$ from the collection of BC.

Keywords: Bloodstream infections, Bacteremia, Antibiotic resistance, Carbapenem-resistant, Carbapenemases, KPC, Klebsiella pneumoniae, Mortality, Time to appropriate antibiotic therapy

\footnotetext{
* Correspondence: marco.falcone@unipi.it

'Infectious Diseases Unit, Azienda Ospedaliera Universitaria Pisana, University

of Pisa, Via Paradisa, 2, 56124 Pisa, PI, Italy

Full list of author information is available at the end of the article
}

(c) The Author(s). 2020 Open Access This article is distributed under the terms of the Creative Commons Attribution 4.0 International License (http://creativecommons.org/licenses/by/4.0/), which permits unrestricted use, distribution, and reproduction in any medium, provided you give appropriate credit to the original author(s) and the source, provide a link to the Creative Commons license, and indicate if changes were made. The Creative Commons Public Domain Dedication waiver (http://creativecommons.org/publicdomain/zero/1.0/) applies to the data made available in this article, unless otherwise stated. 


\section{Introduction}

The spread of Klebsiella pneumoniae carbapenemase (KPC)-producing Klebsiella pneumoniae (Kp) is an urgent public health threat requiring immediate action $[1,2]$. As a matter of fact, carbapenem-resistant (CR) Kp infections are associated with high attributable mortality (2118 attributable deaths out of a total of 15,947 infections), and with a median number of total disability-adjusted life years of 11.5 per 100,000 population [3]. Mortality rates of patients with bloodstream infections (BSIs) caused by KPC-Kp range from 40 to $50 \%[4,5]$.

Treatment of patients with BSIs caused by KPC-Kp represents a challenge for clinicians, and no data from randomized clinical trials comparing different antibiotic strategies are currently available [6]. Observational studies indicate that combination therapies are more effective than monotherapies $[7,8]$. Moreover, a retrospective study performed in intensive care unit (ICU) patients with septic shock showed that an antibiotic regimen containing at least two in vitro active antibiotics is associated with improved survival [9]. Novel drugs represent new promising treatment options, but larger studies are needed to evaluate their impact on survival of patients with KPC-Kp infections [10-12]. Thus, the optimal therapy of KPC-Kp BSI has not been defined.

Timely and appropriate antimicrobial therapy is critically important for treatment of patients with BSI [13, 14]. Recent studies highlighted that inappropriate empiric treatment is associated with poor outcome of patients with urinary tract [15] or any type of infection due to KPC-Kp $[16,17]$. Among non-neutropenic patients with BSI due to KPC-Kp, Satlin et al. did not find any association between timing of active therapy and mortality, but a trend to higher mortality rates was observed in patients who did not receive active therapy within the first $12 \mathrm{~h}$ from BSI onset [18]; however, the majority of patients (84\%) acquired infection in the community or in non-ICU wards [18]. Thus, the relationship between timing of appropriate therapy and outcome of BSI caused by KPC-Kp has not been specifically investigated in ICU patients.

The aim of this study was to assess the relationship between the time to appropriate antibiotic therapy and the clinical outcome in ICU patients with BSI caused by KPC-Kp.

\section{Methods}

\section{Study design and data collection}

This observational retrospective study was conducted from January 2015 to December 2018 in two University tertiary care hospitals: Azienda Ospedaliera Universitaria Pisana (Pisa, Italy) and Santa Maria della Misericordia Hospital (Udine, Italy). All consecutive adult patients with a BSI due to KPC-Kp hospitalized in ICU were included in the study. Patients with polymicrobial BSIs were excluded. The study was conducted according to the principles stated in the Declaration of Helsinki.

Data were extracted from the medical records of patients and from hospital computerized databases. The following data were collected: demographics, clinical and laboratory findings, comorbidities, microbiologic data, source of infection, and source control. All patients underwent screening rectal swab at admission in the ICU and twice weekly. Data about rectal colonization by KPC-Kp were recorded. Sequential Organ Failure Assessment (SOFA) score at time of BSI and APACHE II score were calculated $[19,20]$. Data about hospitalization and antibiotic therapy in the previous 90 days before the BSI were also reviewed. Treatment regimens were analyzed and time from collection of blood cultures to administration of appropriate antibiotic therapy was calculated and reported in hours.

\section{Outcome and assessments}

The primary outcome of the study was the relationship between the time to appropriate antibiotic therapy and 30-day mortality. Time to appropriate antibiotic therapy was defined as the time (in hours) from blood culture collection and the administration of in vitro active antibiotic therapy. In order to evaluate the impact of time to appropriate antibiotic therapy on 30-day mortality, time was assessed as categories of 24-h increments.

The secondary outcome was to evaluate if different treatment regimens influence the 30-day mortality and a composite endpoint of mortality or nephrotoxicity (postbaseline increase in serum creatinine $>1.0 \mathrm{mg} / \mathrm{dL}$ or adverse events preferred term of renal failure, renal failure acute, or renal impairment). To this end, we identified three groups of treatment: (1) colistin-containing regimen, (2) ceftazidime/avibactam ( \pm fosfomycin or aminoglycosides), (3) other regimens.

\section{Definitions}

A KPC-Kp BSI was defined as a BSI documented by blood culture positivity for a KPC-Kp strain. According to the National Healthcare Safety Network, the probable source of BSI was assessed according to the available clinical and microbiological information and classified by the investigators using the following categories: BSI from central venous catheter (CVC), respiratory tract infection, urinary tract infection (UTI), skin and skin structure infection (SSTI), intra-abdominal infection (IAI), endocarditis, and primary BSI in the absence of an identified source of infection growing the same organism as recovered from blood [21]. The CVC was considered a likely source of infection if the blood culture obtained from the lumen of the catheter was positive at a time $<2 \mathrm{~h}$ compared to peripheral veins, and/or culture of the catheter was positive. 
BSI onset was defined as the date of collection of the index blood culture. Time to adequate antibiotic therapy was defined as the number of hours from blood culture collection and the administration of active antibiotic therapy. Control of removable source of infection was defined as removal of any preexisting contaminated $\mathrm{CVC}$ and as drainage of intra-abdominal abscesses or other fluid collections thought to be the source of KPC$\mathrm{Kp}$ infection within $24 \mathrm{~h}$ from the onset of BSI. Cardiovascular disease was defined as the presence of one or more of the following conditions: previous coronary heart disease, peripheral arterial disease, heart failure, paroxysmal atrial fibrillation, and chronic (persistent or permanent) atrial fibrillation [22].

BSI episodes were classified as nosocomial, healthcareassociated, and community-acquired according to previous definitions [23]. Nosocomial BSIs were defined by positive blood cultures obtained from patients who had been hospitalized for $48 \mathrm{~h}$ or longer; health careassociated BSIs were defined by positive blood cultures obtained from a patient at the time of hospital admission or within $48 \mathrm{~h}$ of admission if the patient fulfilled any of the following criteria: received intravenous therapy at home, received wound care or specialized nursing care or had self-administered intravenous medical therapy in the 30 days before the BSI, attended a hospital or hemodialysis clinic or received intravenous chemotherapy in the 30 days before the BSI, was hospitalized in an acute care hospital for two or more days in the 90 days before the BSI, or was a resident in a nursing home or long-term care facility. Community-acquired BSI was defined by a positive blood culture obtained at the time of hospital admission or within the $48 \mathrm{~h}$ after hospital admission for patients who did not fit the criteria for a health care-associated infection [23]. The presence of septic shock was defined according to the last proposed criteria (Sepsis-3) [24]. Information about specific causes of death was collected. Cause of death was defined as the primary pathology (irrespective of its duration) leading to death of the patient and was classified as previously reported [25].

\section{Antimicrobial treatment evaluation}

All patients were evaluated by a dedicated infectious disease specialist throughout their entire ICU hospitalization. Empiric antibiotic therapy was selected according to clinical judgment by infectious disease specialists, which was subsequently modified according to blood cultures results. According to internal ICU protocols, tigecycline was administered at high dosages (100 mg iv q12h), and ß-lactams, $ß$-lactamase inhibitors, and carbapenems were administered using extended infusion (meropenem over 4$6 \mathrm{~h}$ ). According to local ICU protocols, colistin dosages were uniformly administered at the dosages of 9 million
IU daily in patients with normal renal function. Modification of dosage schedules based on renal function was performed according to indications from the Food and Drug Administration [26].

Antibiotic treatment was classified as appropriate if at least one administered antibiotic displayed documented in vitro susceptibility according to the breakpoints established by the European Committee on Antimicrobial Susceptibility Testing (EUCAST). Combination therapy was defined as treatment regimens including two or more in vitro active antibiotics, administered at the same time. Antibiotic regimens were also analyzed according to the following classification: no antibiotic displaying in vitro activity, only one antibiotic displaying in vitro activity, and two or more antibiotics displaying in vitro activity.

\section{Microbiology and KPC identification}

From positive blood cultures, Gram staining and a rapid identification protocol were adopted [27]. The bacterial pellet obtained directly from positive blood cultures was used for MALDI-TOF MS (Bruker Daltonics) identification and for molecular analysis. The presence of a bla $\mathrm{KPC}_{\mathrm{KPC}}$ gene was determined by PCR using the GeneXpert ${ }^{\circ}$ System (Cepheid). Antimicrobial susceptibility tests were performed with the SensiTitre ${ }^{\mathrm{m}}$ system (Thermo Fisher Scientific) or Vitek 2 automated system (bioMérieux, Marcy l'Etoile, France) according to the manufacturer's instructions. Minimum inhibitory concentrations (MICs) were classified according to breakpoints established by the European Committee on Antimicrobial Susceptibility Testing (EUCAST) [28].

\section{Statistical analysis}

Continuous variables were reported as mean \pm standard deviation and medians and interquartile ranges (IQRs) according to their distribution. The Student $t$ test was used when comparing normally distributed data, and the Mann-Whitney $U$ test was used to analyze non-normally distributed data. Categorical data were expressed as frequency distributions, and the chi-square test or Fisher exact test was used to determine if differences existed between groups.

According to the primary outcome of the study, a Cox regression analysis was performed to determine the effects of different variables on the overall 30-day mortality and on the 30-day mortality specifically due to infection. Multivariate analysis using logistic regression prediction models was constructed using a forward stepwise procedure, entering all variables with univariate $p<0.05$ and those deemed clinically significant. Age, SOFA score, and time from blood culture collection to appropriate antibiotic therapy were continuous (24-h increments were considered for time to appropriate therapy). Potential 
baseline confounders, including diabetes, cardiovascular disease, COPD, and primary bacteremia were considered for the multivariate analysis. The final multivariate model was chosen according to the Akaike information criterion and to parsimony and clinical interpretability of data. Kaplan-Meier curves were constructed to compare time to 30-day mortality between patients who received appropriate antibiotic therapy within the first $24 \mathrm{~h}$ from the blood cultures collection and those who did not. Moreover, a more formal causal analysis was conducted based on propensity scores (PS). Propensity scores were estimated using a logistic regression model to predict time to adequate therapy $<24 \mathrm{~h}$ using age, diabetes, Charlson Comorbidity index, and SOFA score. Balance tables were used to assess adequacy of matching via propensity score. Estimated propensity scores were then used to perform greedy matching without replacement. The matched subset was then used to estimate the relationship between time to adequate therapy and the endpoints using Cox regression. We evaluated both univariate Cox regression models and models including a random effect specific to each matched couple (conditional Cox regression), whose results did not differ substantially.

A sensitivity analysis was performed to evaluate the effect of combination therapy (defined as above-reported) on 30-day mortality. A forest plot was used to describe the results of the sensitivity analysis.

To evaluate differences in clinical outcome among treatment regimens, differences in 30-day mortality and in the composite endpoint of 30-day mortality or nephrotoxicity were analyzed using the Cox analysis. Colistin-containing regimen was selected as reference variable; both ceftazidime-avibactam-containing regimen and other regimens were tested against the reference variable. Patients who did not receive any in vitro active therapy was excluded. Hazard ratio (HR) and 95\% confidence intervals (CIs) were calculated to evaluate the strength of any association. Statistical significance was established at $p \leq 0.05$. All reported $p$ values are two tailed. The results obtained were analyzed using a commercially available statistical software package (SPSS 20.0; IBM, Armonk, NY, USA and R 3.5.1, Vienna, Austria).

\section{Results}

\section{Study population}

Among 114 ICU patients with Kp-KPC BSI, a total of 102 were included in the study. Flowchart of the study is shown in Fig. 1. Overall, median age was 64 years (IQR 53-74). The most common source of infection was IAI (23.5\%), followed by UTI (20.6\%), SSTI (17.6\%), CVC (12.7\%), and pneumonia (10.8\%). Fourteen (13.7\%) patients had a primary BSI. The majority of patients had recorded previous rectal colonization by KPC-Kp (51\%).

Septic shock occurred in $39.2 \%$ of patients. The 7-day, 14-day, and 30-day mortality rates were $20.6 \%, 32.4 \%$, and $45 \%$, respectively. All deaths occurred during the ICU stay. Among 46 non-surviving patients, 43 (93.5\%) died due to infection, 2 patients (4.3\%) from acute hemorrhage, and 1 (2.2\%) from acute respiratory failure. The baseline characteristics of the study population and the univariate analysis of patients by survival are reported in Table 1. Compared to surviving patients, those who died had a higher median age (70.5 [IQR 60-77.25] vs 55 years [IQR $41.5-70.75], p<0.001)$ were more likely

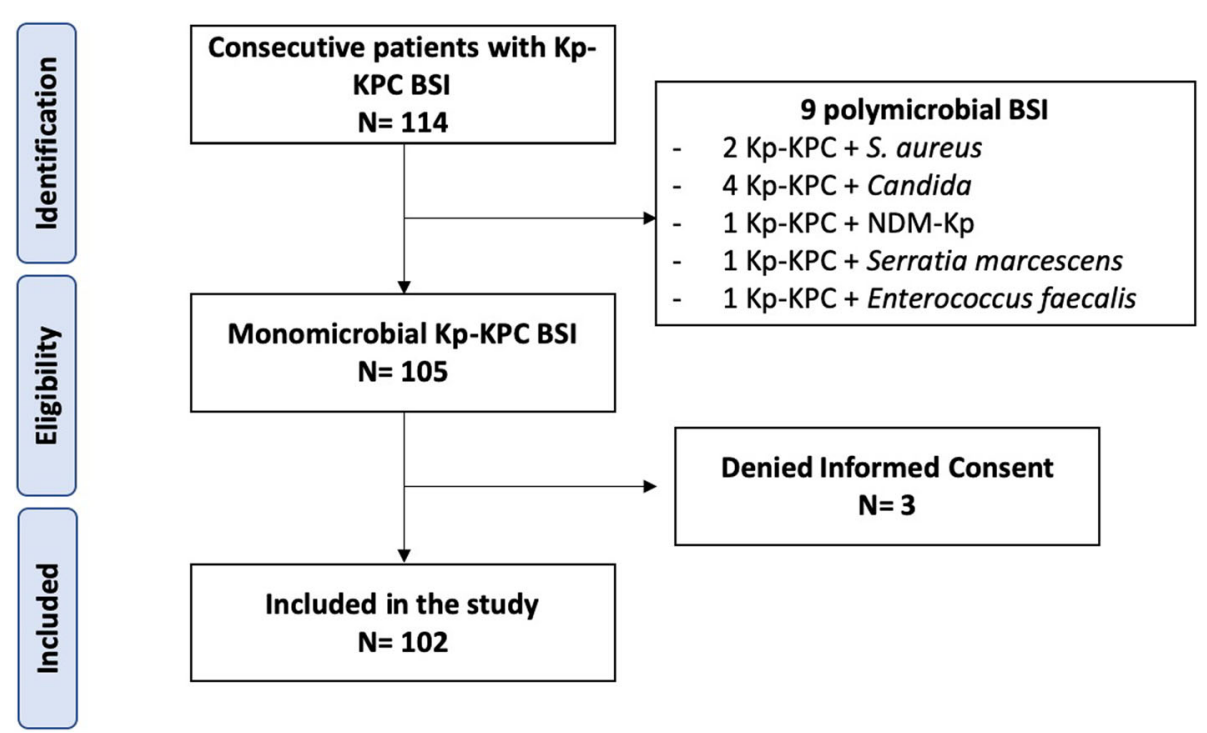

Fig. 1 Study flow chart. BSI bloodstream infections, Kp-KPC Klebsiella pneumoniae carbapenemase-producing Klebsiella pneumoniae, NDM New-Delhi-metallo-beta-lactamase 
Table 1 Baseline characteristics of population and univariate analysis of patients with KPC-Kp BSI by survival status

\begin{tabular}{|c|c|c|c|c|}
\hline Characteristic & $\begin{array}{l}\text { All patients } \\
N=102\end{array}$ & $\begin{array}{l}\text { Patients who } \\
\text { survived } \\
N=56\end{array}$ & $\begin{array}{l}\text { Patients who did } \\
\text { not survive } \\
N=46\end{array}$ & $p$ value \\
\hline Age, years, median (IQR) & $64(53-74)$ & 55 (41.5-70.75) & $70.5(60-77.25)$ & $<0.001$ \\
\hline Male sex & $65(63.7 \%)$ & 39 (69.6\%) & $26(56.5 \%)$ & 0.170 \\
\hline \multicolumn{5}{|l|}{ Comorbidities } \\
\hline Diabetes & $18(17.6 \%)$ & $5(8.9 \%)$ & $13(28.3 \%)$ & 0.011 \\
\hline Cardiovascular disease & $36(35.3 \%)$ & $11(19.6 \%)$ & $25(54.3 \%)$ & $<0.001$ \\
\hline Chronic renal disease & $15(14.7 \%)$ & $6(10.7 \%)$ & $9(19.6 \%)$ & 0.209 \\
\hline Chronic liver disease & $13(12.7 \%)$ & $9(16.1 \%)$ & $4(8.7 \%)$ & 0.266 \\
\hline COPD & $10(9.8 \%)$ & $2(3.6 \%)$ & $8(17.4 \%)$ & 0.020 \\
\hline Solid cancer & 27 (26.5\%) & $13(23.2 \%)$ & $14(30.4 \%)$ & 0.411 \\
\hline Hematological malignancy & $14(13.7 \%)$ & $8(14.3 \%)$ & $6(13 \%)$ & 0.856 \\
\hline Solid organ transplantation & $8(7.8 \%)$ & $5(8.9 \%)$ & $3(6.5 \%)$ & 0.653 \\
\hline Previous hospitalization & 40 (39.2\%) & $21(37.5 \%)$ & $19(41.3 \%)$ & 0.695 \\
\hline Previous antibiotic therapy & $58(56.9 \%)$ & $35(62.5 \%)$ & $23(50 \%)$ & 0.205 \\
\hline Length of ICU stay after KPC-Kp BSI, days, median (IQR) & $18(9-28.5)$ & $24(15.75-44.75)$ & $12(5-18)$ & $<0.001$ \\
\hline ICU stay, days, median (IQR) & $39(21-59)$ & $44.5(29.75-75.5)$ & $27(15.5-50.75)$ & $<0.001$ \\
\hline Hospital length of stay before bacteremia, days, median (IQR) & $17.5(5.75-36.5)$ & $18.5(7-34.25)$ & $13(3.75-39)$ & 0.362 \\
\hline Primary cause of ICU admission & $16(15.7 \%)$ & $8(14.3 \%)$ & $8(17.4 \%)$ & 0.668 \\
\hline Trauma & $16(15.7 \%)$ & $11(19.6 \%)$ & $5(10.9 \%)$ & 0.225 \\
\hline Respiratory failure & $16(15.7 \%)$ & $12(21.4 \%)$ & $4(8.7 \%)$ & 0.078 \\
\hline Cardiovascular disease & $15(14.7 \%)$ & $6(10.7 \%)$ & $9(19.6 \%)$ & 0.209 \\
\hline Surgery & $13(12.7 \%)$ & $7(12.5 \%)$ & $6(13 \%)$ & 0.935 \\
\hline Infection & $11(10.8 \%)$ & $6(10.7 \%)$ & $5(10.9 \%)$ & 0.980 \\
\hline Burn injury & $10(9.8 \%)$ & $3(5.4 \%)$ & $7(15.2 \%)$ & 0.096 \\
\hline Cerebrovascular accident & $5(4.9 \%)$ & $3(5.4 \%)$ & $2(4.3 \%)$ & 0.814 \\
\hline \multicolumn{5}{|l|}{ Other* } \\
\hline \multicolumn{5}{|l|}{ Source of infection } \\
\hline CVC-related bacteremia & $13(12.7 \%)$ & $11(19.6 \%)$ & $2(4.3 \%)$ & 0.021 \\
\hline Primary bacteremia & $14(13.7 \%)$ & $2(3.6 \%)$ & $12(26.1 \%)$ & 0.001 \\
\hline Respiratory tract & $11(10.8 \%)$ & $6(10.7 \%)$ & $5(10.9 \%)$ & 0.980 \\
\hline Urinary tract & $21(20.6 \%)$ & $12(21.4 \%)$ & $9(19.6 \%)$ & 0.817 \\
\hline Skin and skin structure & $18(17.6 \%)$ & $13(23.2 \%)$ & $5(10.9 \%)$ & 0.104 \\
\hline Intra-abdominal & $24(23.5 \%)$ & $12(21.4 \%)$ & $12(26.1 \%)$ & 0.581 \\
\hline Endocarditis & $1(0.9 \%)$ & 0 & $1(2.2 \%)$ & 0.268 \\
\hline \multicolumn{5}{|l|}{ Type of acquisition } \\
\hline Healthcare-associated & $13(12.7 \%)$ & $3(5.4 \%)$ & $10(21.7 \%)$ & 0.014 \\
\hline Nosocomial & 89 (87.2\%) & $53(94.6 \%)$ & $36(78.3 \%)$ & 0.014 \\
\hline Charlson Comorbidity Index, median (IQR) & $2(1-3)$ & $2(0-3)$ & $2(1.75-3.25)$ & 0.078 \\
\hline KPC-Kp intestinal colonization & $52(51 \%)$ & $31(55.4 \%)$ & $21(45.7 \%)$ & 0.329 \\
\hline Source control & $65(63.7 \%)$ & $40(81.6 \%)$ & $25(80.6 \%)$ & 0.912 \\
\hline Septic shock & $40(39.2 \%)$ & $18(32.1 \%)$ & $22(47.8 \%)$ & 0.106 \\
\hline Mechanical ventilation & $40(39.2 \%)$ & $23(41.1 \%)$ & $17(37 \%)$ & 0.672 \\
\hline AKI & $15(14.7 \%)$ & $8(14.3 \%)$ & $7(15.2 \%)$ & 0.895 \\
\hline SOFA score, median (IQR) & $5(3-9.5)$ & $4(2-10)$ & $7.5(4-9.25)$ & 0.034 \\
\hline
\end{tabular}


Table 1 Baseline characteristics of population and univariate analysis of patients with KPC-Kp BSI by survival status (Continued)

\begin{tabular}{|c|c|c|c|c|}
\hline Characteristic & $\begin{array}{l}\text { All patients } \\
N=102\end{array}$ & $\begin{array}{l}\text { Patients who } \\
\text { survived } \\
N=56\end{array}$ & $\begin{array}{l}\text { Patients who did } \\
\text { not survive } \\
N=46\end{array}$ & $p$ value \\
\hline APACHE II score, median (IQR) & $15(11-21)$ & $13.5(10-20)$ & $19(12-21)$ & 0.040 \\
\hline DNR & $3(2.9 \%)$ & $1(1.8 \%)$ & $2(4.3 \%)$ & 0.446 \\
\hline $\begin{array}{l}\text { Time from blood culture collection to appropriate antibiotic } \\
\text { therapy, hours, median (IQR) }\end{array}$ & $15(1-60)$ & $8.5(1-36)$ & $48(5-108)$ & 0.014 \\
\hline
\end{tabular}

$A K I$ acute kidney injury, $B S I$ bloodstream infection, $C O P D$ chronic obstructive pulmonary disease, $C V C$ central venous catheter, $D N R$ do not resuscitate order, ICU intensive care unit, IQR interquartile range, KPC-Kp KPC-producing Klebsiella pneumoniae, SOFA Sequential Organ Failure Assessment. Statistical significant $p$ values are indicated in italics

* Other causes of ICU admission include 1 carbon monoxide poisoning, 1 Sezary syndrome, 1 acute renal failure, 1 Wilson disease with hepatic failure,

1 thyrotoxicosis

to be affected by diabetes mellitus $(28.3 \%$ vs $8.9 \%, p=$ $0.011)$, cardiovascular disease $(54.3 \%$ vs $19.6 \%, p<$ 0.001 ), and chronic obstructive pulmonary disease $(17.4 \%$ vs $3.6 \%, p=0.020)$, and had a higher median SOFA score (7.5 [IQR 4-9.25] vs 4 [IQR 2-10], $p=$ 0.034). CVC-related bacteremia was more frequent among survivors $(19.6 \%$ vs $4.3 \%, p=0.021)$, while a primary BSI was more common among patients who died (26.1\% vs $3.6 \%, p=0.001)$. Three patients of 87,88 , and 90 years old, respectively, with multiple comorbidities received a "do not resuscitate order (DNR); one of them had severe burn injury.

Susceptibility rates of the $102 \mathrm{KPC}-\mathrm{Kp}$ isolates are shown in Additional file 1: Table S1. The antibiotic regimens used for KPC-Kp BSIs in patients by survival status are shown in Additional file 1: Table S2. The most frequent antibiotic regimen was colistin plus meropenem plus tigecycline \pm gentamycin (59.8\%), followed by ceftazidime-avibactam alone or in combination with fosfomycin or aminoglycosides (12.7\%) and other regimens (16.7\%). Among 52 patients receiving a combination therapy including meropenem, MIC of the infecting strain was $<8 \mathrm{mg} / \mathrm{L}$ in $3.8 \%$ of cases, equal to $8 \mathrm{mg} / \mathrm{L}$ in $7.7 \%$, ranging from 16 to $64 \mathrm{mg} / \mathrm{L}$ in $48.1 \%$, and more than $64 \mathrm{mg} / \mathrm{L}$ in $40.4 \%$. A prolonged infusion of antibiotics was used in 46 (88.5\%) patients receiving meropenem and in 2 (15.4\%) patients receiving CAZ-AVI. The proportion of patients not receiving in vitro active therapy was higher for those who died (19.6\%) compared with those who survived (3.6\%, $p=0.010)$. Two patients with CVC-related BSI not receiving any active antibiotic survived after a prompt removal of the infected device. Nine patients did not receive any antibiotic active in vitro and all of them died during the 30 days following bacteremia: 5 of them did not receive any antibiotic therapy because they died during the first $24 \mathrm{~h}$ from blood culture collection; 4 of them received a regimen containing colistin or tigecycline, but were affected by BSI due to pan drug-resistant Kp.

\section{Primary outcome analysis}

The median time from blood culture collection to the start of appropriate antibiotic therapy was significantly shorter in surviving patients $(8.5 \mathrm{~h}$ [IQR $1-36])$ than in patients who died (48 h [IQR 5-108], $p=0.014$ ). Among patients with previous rectal colonization by KPC-Kp, time from blood culture collection to appropriate antibiotic therapy was significantly shorter than that observed among non-colonized patients (5 h [IQR 0-49] vs 23.5 h [IQR 6-108.5], $p=0.004$ ).

Figure 2 shows 30-day mortality rates according to different categories of time from blood culture collection to appropriate antibiotic therapy. Patients appropriately treated within the first $24 \mathrm{~h}$ (see Additional file 1: Table S3) had lower 30-day mortality rates $(29.1 \%$ vs $63.8 \%$, $p<0.001)$. Out of 52 patients receiving meropenem therapy, there were no significant differences in the distribution of MICs between patients receiving early (within 24 h) or late $(>24 \mathrm{~h})$ appropriate therapy. After the exclusion of the 9 patients who did not receive any antibiotic active in vitro, 30-day mortality rates remained higher in patients who did not receive active antibiotic therapy compared to those who receive an appropriate antibiotic therapy within the first $24 \mathrm{~h}$ from the blood culture collection (58.3\% versus 29.1\%, $p=0.005)$. Figure 3 shows the Kaplan-Meier survival curves for patients who received or not an appropriate antibiotic therapy within $24 \mathrm{~h}$ from blood culture collection. A PS-based matched analysis was performed: we were able to match 47 couples to patients who received or not in vitro active therapy within $24 \mathrm{~h}$ from blood culture collection. The matched patients did not show significant differences in exposure to variables related to early antibiotic therapy. Conditional logistic regression in PS-matched cohorts showed that in vitro active therapy within $24 \mathrm{~h}$ from blood culture collection was associated with lower 30-day mortality $\quad(\mathrm{HR}=0.36, \quad 95 \% \mathrm{CI}: \quad 0.188-0.690$, $p=0.0021)$.

Factors independently associated with the overall 30day mortality, by Cox regression analysis, were primary 


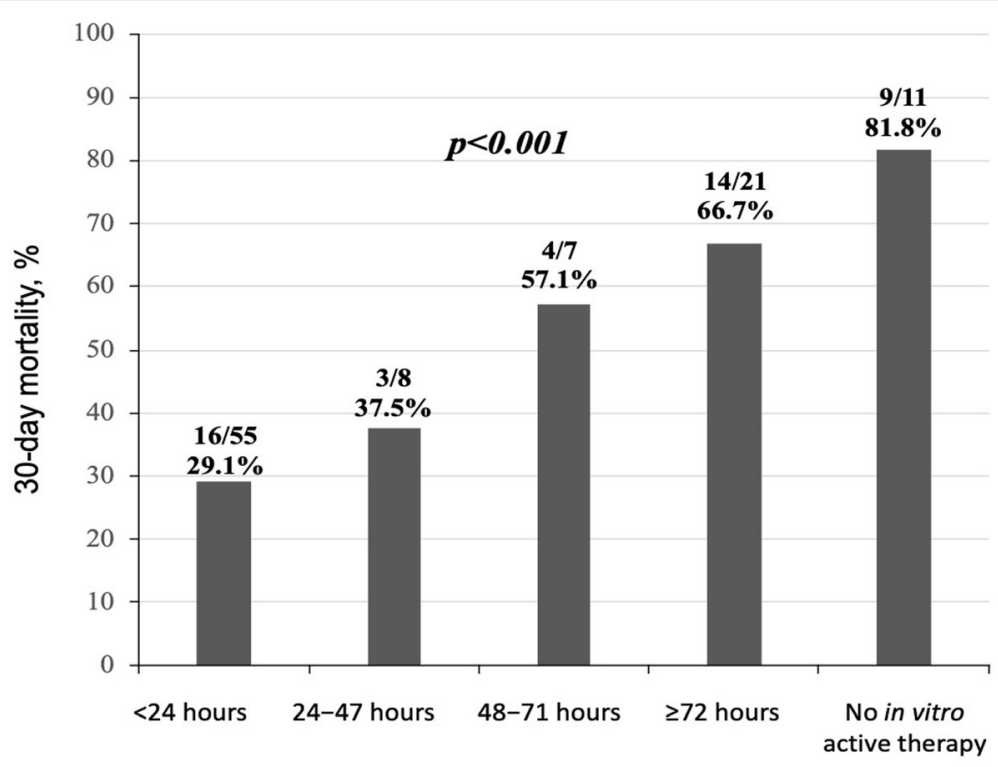

Time from blood culture to appropriate therapy

Fig. 2 Thirty-day mortality rates by time from blood culture collection to appropriate antibiotic therapy

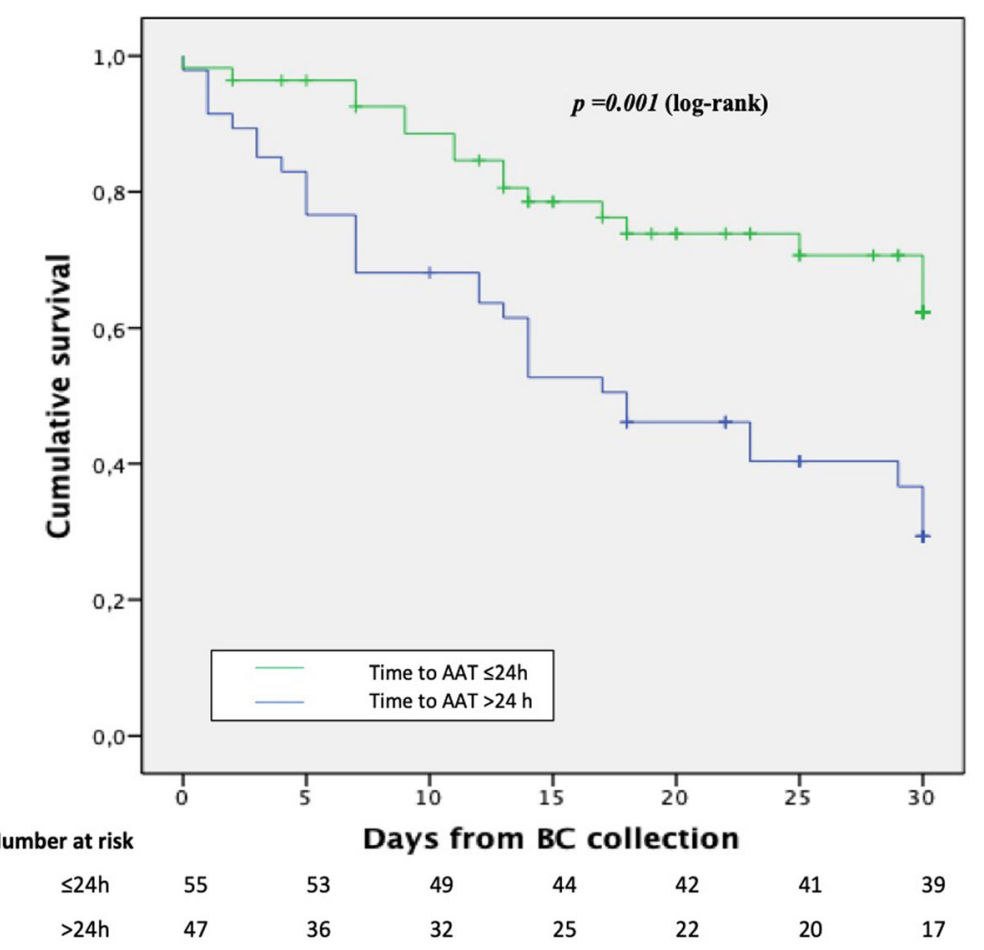

Fig. 3 Kaplan-Meier for 30-day survival according to the receipt of appropriate antibiotic therapy within the first $24 \mathrm{~h}$ from blood culture collection. AAT appropriate antibiotic therapy; BC blood cultures 
bacteremia (HR 2.662 [95\% CI 1.118-6.336], $p=0.027$ ) cardiovascular disease (HR 2.196 [95\% CI 1.082-4.457], $p=0.029$ ), time (24-h increments) from blood culture collection to appropriate therapy (HR 1.382 [95\% CI 1.132-1.687], $p=0.001$ ), SOFA score (HR 1.122 [95\% CI 1.036-1.216], $p=0.005$ ), and age (HR 1.030 [95\% CI 1.006-1.054], $p=0.012$ ) (Table 2). Time from blood culture collection to appropriate antibiotic therapy was independently associated with 30-day mortality, even excluding patients who received only tigecycline as active antibiotic. The sensitivity analysis showed that combination therapy with two or more in vitro active antibiotics was not associated with increased 30-day mortality (HR $1.58,95 \%$ CI $0.69-3.62, p=0.280$ ). Figure 4 shows the forest plot of the adjusted HR of each factor included in the sensitivity analysis.

\section{Secondary outcome analysis}

Table 3 shows the comparison of 30-day mortality and the composite endpoint according to treatment regimens. No treatment regimen influenced the 30-day mortality. Instead, CAZ-AVI-containing regimens were associated with reduced risk of composite endpoint (30day mortality OR nephrotoxicity) (HR 0.231 [95\% CI 0.071-0.745], $p=0.014$ ) compared to colistin-containing regimens.

Additional file 1: Figure S1 shows the comparison of 30-day mortality among the three groups of antibiotic regimens stratified by site of infections. The higher 30day mortality was observed in subjects with primary bacteremia (81.8\%), followed by IAI (50\%) and respiratory tract $(33.3 \%)$, while the least mortality rate was observed in patients with CVC-related bacteremia (18\%, $p=0.009$ ANOVA test). In patients with primary bacteremia, mortality rate was higher in patients who received colistin-based regimen compared to those who received other antibiotic regimens (100\% vs 50\% $p=0.039$ ). There were no differences in antibiotic dosages among patients with different site of infections.

\section{Discussion}

This study demonstrates that time from blood culture collection to appropriate antibiotic therapy is independently associated with clinical outcome for ICU patients with BSI due to $\mathrm{KPC}-\mathrm{Kp}$, even after adjustment for comorbidities, severity of illness and age.

Delayed administration of appropriate antibiotic therapy is associated with high mortality rates in patients with sepsis or septic shock [29-32], and the probability of death increases with the number of hours of delay of antibiotic administration [31]. Moreover, time from blood culture collection to the administration of appropriate antibiotic therapy also influences the length of hospital stay [33]. No studies specifically evaluated the impact of the timing of appropriate antibiotic therapy in ICU patients with BSI by KPC-Kp. Our findings are unique in the population studied and could influence the future management of ICU patients with BSI due to KPC-Kp.

Our study demonstrated that the administration of appropriate antibiotic therapy within $24 \mathrm{~h}$ of blood culture collection is associated with 30-day mortality rates lower than $30 \%$. Conversely, the risk of death progressively increased when appropriate antibiotic therapy was started 24-48 h (mortality rate $37.5 \%$ ), 48-72 h (mortality rate $57.1 \%$ ), and $>72 \mathrm{~h}$ (mortality rate $66.7 \%$ ) from the index blood culture collection. Based on these results, the identification of patients with a high probability of BSI caused by KPC-Kp represents a great challenge for clinicians.

In the clinical practice, the median turnaround time from specimen collection to antimicrobial susceptibility testing results is 2.71 days [34]. In our study, this time is associated with increased mortality. Thus, it appears crucial to deliver effective therapy against KPC-Kp as soon as possible, and specifically within the first $24 \mathrm{~h}$ from the collection of blood cultures. A range of strategies may be useful in achieving this goal. According to the study results, our hospitals implemented the following recommendations for ICU patients: (i) regular rectal screening

Table 2 Cox regression analysis of factors associated with 30-day mortality in patients with KPC-Kp BSI

\begin{tabular}{lll}
\hline & $H R(95 \% \mathrm{Cl})$ & $p$ value \\
\hline Primary bacteremia & $2.662(1.118-6.336)$ & 0.027 \\
Cardiovascular disease & $2.196(1.082-4.457)$ & 0.029 \\
Time from blood culture collection to appropriate therapy (24-h increments) & $1.382(1.132-1.687)$ & 0.001 \\
SOFA score (1-point increments) & $1.122(1.036-1.216)$ & 0.005 \\
Age (1-year increments) & $1.030(1.006-1.054)$ & 0.012 \\
Diabetes & $1.960(0.793-4.849)$ & 0.145 \\
COPD & $1.303(0.513-3.306)$ & 0.578 \\
\hline
\end{tabular}

BSI bloodstream infection, $\mathrm{Cl}$ confidence interval, HR hazard ratio, KPC-Kp KPC-producing Klebsiella pneumoniae, SOFA Sequential Organ Failure Assessment. Statistical significant $p$ value are indicated in italics 


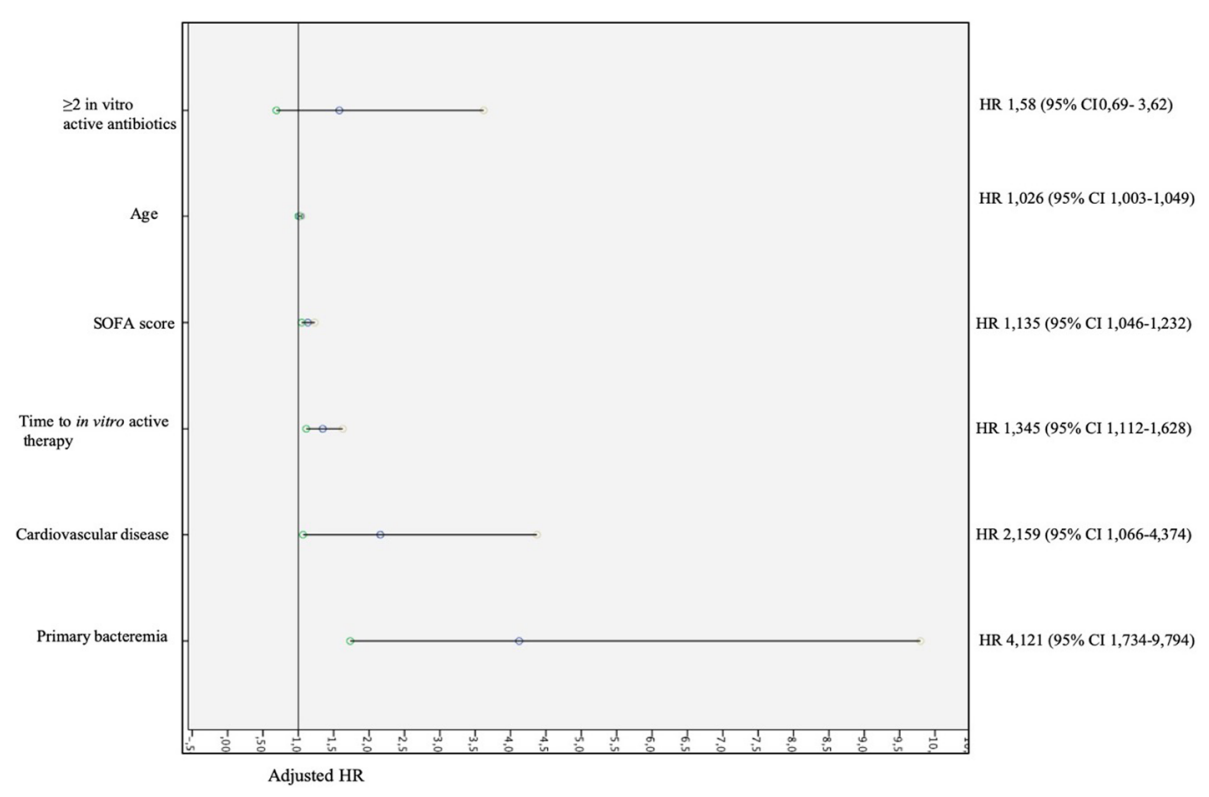

Fig. 4 Forest plot showing adjusted HR of factors associated with 30-day mortality included in the sensitivity analysis

in all hospitalized patients to early identify KPC-Kp rectal carriers, (ii) starting an empirical therapy targeting $\mathrm{KPC}-\mathrm{Kp}$ in rectal carriers with septic syndrome and contemporary presence of additional risk factors for KPC-Kp bacteremia (recent abdominal invasive procedure, receipt of chemotherapy/radiation therapy, additional colonization sites; a Giannella risk score $\geq 7$ justify the administration of antibiotics covering KPC-Kp [35]), (iii) prompt deescalation in case of not confirmed KPC-Kp infection as soon as microbiological results are available. This approach may be reasonable in endemic settings for KPC-Kp but cannot be generalized to other settings. New rapid diagnostic techniques enabling fast identification of genes coding for carbapenemase enzymes [36, 37] will be useful to improve this clinical strategy.

We found no differences in 30-day mortality rates among patients who received colistin-base, CAZ-AVI- based, or other treatment regimens. However, the composite endpoint of 30-day mortality or nephrotoxicity demonstrated a superior risk benefit profile for CAZ-AVI compared to colistin-containing regimens $(p=0.01)$. This is in line with recent reports showing that treatment-related nephrotoxicity was significantly higher in patients treated with colistin [38-40].

Furthermore, we were able to identify differences in mortality according to the site of infection. Patients with primary bacteremia and those with IAIs had the highest mortality rates, while patients with CVC-related BSI had the least mortality $(81.8 \%$ vs $50 \%$ vs $18 \%$, respectively $p=0.009$ ); a large percentage of patients with primary bacteremia and IAI received colistin (63.6 and 37.5\%, respectively) and the mortality in patients treated with colistin was higher than comparators. Although not statistically significant, this trend was observed also in

Table 3 Comparison of 30-day mortality and exploratory endpoint according to treatment regimens

\begin{tabular}{|c|c|c|c|c|}
\hline & Antibiotic regimens & N (\%) & HR $(95 \% \mathrm{Cl})$ & $p$ value \\
\hline \multicolumn{5}{|l|}{ 30-day mortality } \\
\hline & COL containing-regimen & 27/61 (44.3\%) & Ref variable & Ref variable \\
\hline & CAZ-AVI containing-regimen & $3 / 13(23.1 \%)$ & $0.424(0.129-1.391)$ & 0.157 \\
\hline & Other regimens & $7 / 17(41.2 \%)$ & $0.799(0.366-1.746)$ & 0.574 \\
\hline \multicolumn{5}{|l|}{ Composite endpoint } \\
\hline \multirow[t]{3}{*}{ 30-day mortality OR nephrotoxicity } & COL containing-regimen & 42/61 (68.9\%) & Ref variable & Ref variable \\
\hline & CAZ-AVI containing-regimen & $3 / 13(23.1 \%)$ & $0.231(0.071-0.745)$ & 0.014 \\
\hline & Other regimens & $8 / 17(47.1 \%)$ & $0.503(0.245-1.034)$ & 0.061 \\
\hline
\end{tabular}


patients with BSI arising from an IAI (64.3\% vs $37.5 \%)$ and CVC-related bacteremia (37.5\% vs 0\%). A recent study showed that in patients with post-surgical IAI due to carbapenem-resistant Acinetobacter baumannii adjunctive colistin to conventional tigecycline did not yield clinical benefit but caused higher renal complication and unfavorable non-clinical outcomes [41]. It has been also documented that after the administration of a loading dose of colistin, concentration increases more slowly in peritoneal fluid than in plasma [42], and a reduced penetration rate and a potential inoculum effect of colistin in a difficult-toreach site such as the abdominal cavity have been observed [43]. Conversely, despite the small number of cases, in patients with BSI from the urinary tract we observed a lower mortality in those who received colistin-containing regimens compared to other regimens (33.3\% vs $100 \%)$. This may be due to a rapid urinary excretion and higher concentration in the urinary tract of intravenously administered colistin [44]. Unfortunately, the clinical efficacy of colistin therapy is difficult to assess because some routine laboratory methods used for colistin susceptibility testing (in particular gradient tests) have a poor performance [45], and is not ever possible to determine if colistin has appropriate MIC. Thus, reliable microbiological tests and colistin therapeutic drug monitoring should be considered to avoid toxicity and potential clinical consequences.

The observational nature of the study and the small sample size are intrinsic study limitations. Additionally, rather than the clinical diagnosis of BSI, we used blood culture collection time as the point from which to assess timing of appropriate antibiotic therapy, which may introduce a bias in the determination of the timing of appropriate therapy. This bias could potentially have been exacerbated if delays in obtaining blood cultures occurred after suspicion of infection. However, the choice of a welldefined time point allowed us to adopt an objective, standardized criterion. Although rapid identification methods were used, some patients received appropriate therapy after more than $48 \mathrm{~h}$ from BSI onset. It can be occurred because the time to blood culture positivity is variable and can range from few to several hours (or days). Moreover, in our institutions, the microbiology laboratories handle blood cultures 6/7 days weekly, and reporting may be delayed during weekend or public holidays; these factors could explain the prolonged time from blood culture collection to appropriate antibiotic therapy observed in some patients. Anyway, independently from the potential reasons underlining this delay, the time from blood culture collection and the appropriate antibiotic therapy was a factor associated with poor outcome. This finding highlights the crucial role of the microbiology laboratory and the importance of rapid reporting of microbiological results in the management of critically ill patients. Another limitation may be represented by the fact that we considered appropriate an antibiotic therapy if at least one antibiotic, including tigecycline, displayed in vitro activity. Considering its pharmacokinetic profile, the efficacy of tigecycline in patients with BSI due to KPC-Kp is debated and considering it an appropriate therapy may be questionable. However, in our study, we used a high dosage in all cases (100 mg every $12 \mathrm{~h}$ ) and almost all published studies considered tigecycline as an evaluable and potentially effective drug [6]. Future studies should assess the exact role of tigecycline in $\mathrm{KPC}-\mathrm{Kp}$ bacteremia.

\section{Conclusion}

Our study shows that time from blood culture collection to appropriate therapy is an independent predictor of 30-day mortality in patients with KPC-Kp BSI. Clinicians should start appropriate antibiotic therapy as soon as possible, preferably within the first $24 \mathrm{~h}$ of blood culture collection. Treatment strategies allowing the early delivery of in vitro active antibiotics are urgently needed in ICU patients at risk of KPC-Kp bacteremia.

\section{Supplementary information}

The online version of this article (https://doi.org/10.1186/s13054-020-2742-9) contains supplementary material, which is available to authorized users.

Additional file 1: Table S1. In vitro susceptibilities of KPC-producing blood isolates collected from patients hospitalized in ICU. Table S2. Definitive treatment regimens of patients by survival status after a KPC-Kp BSI. Table S3. Comparison between patients who received appropriate antibiotic therapy within the first 24 hours from the blood cultures collection and those who did not. Figure S1. Thirty-day mortality among patients who received different treatment regimens stratified by site of infection. (PDF $167 \mathrm{~kb}$ )

\section{Abbreviations}

BSIs: Bloodstream infections; Cl: Confidence interval; CR: Carbapenemresistant; CVC: Central venous catheter; DNR: Do not resuscitate;

ICU: Intensive care unit; IQRs: Interquartile ranges; Kp: Klebsiella pneumoniae; KPC: Klebsiella pneumoniae carbapenemase; SOFA: Sequential Organ Failure Assessment

\section{Acknowledgements}

Editorial support in the preparation of this article was provided by Linda Townsend, PhD, of Ashfield Healthcare, part of UDG Healthcare plc, and was funded by c\& Co., Ltd., Osaka, Japan.

\section{Authors' contributions}

MF planned and designed the study. EN, ET, AR, and EG collected data. AL, $C G$, and SB performed microbiological analysis. GT performed the statistical analysis. GT and MF drafted the manuscript and designed tables and figures. $M F, M B$, and FM reviewed the final version of the manuscript for intellectual content. All authors read and approved the final manuscript.

\section{Funding}

This study was supported by a grant from Shionogi \& Co., Ltd. (support for medical writing).

\section{Availability of data and materials}

The datasets used and/or analyzed during the current study are available from the corresponding author on reasonable request.

Ethics approval and consent to participate

Local ethical committee (CEAVNO) approved the study. 


\section{Consent for publication}

Not applicable.

\section{Competing interests}

MF received grants and was speaker honoraria for MSD, Angelini, Shionogi. FM has participated in advisory boards and/or received speaker honoraria from Angelini, Correvio, MSD, Pfizer, Astellas, Gilead, BMS, Jansenn, ViiV, BioMerieux, Biotest, Becton-Dickinson, Nordic Pharma, Pfizer, Shionogi. MB has participated in advisory boards and/or received speaker honoraria from Achaogen, Angelini, Astellas, AstraZeneca, Bayer, Basilea, Biomerieux, Cepheid, Cidara, Gilead, Menarini, MSD, Paratek, Pfizer, Roche, The Medicine Company, Shionogi, Tetraphase, VenatoRx and Vifor. The remaining authors declare that they have no competing interests.

\section{Author details}

'Infectious Diseases Unit, Azienda Ospedaliera Universitaria Pisana, University of Pisa, Via Paradisa, 2, 56124 Pisa, PI, Italy. ${ }^{2}$ Infectious Diseases Clinic Department of Health Science, University of Genoa and Hospital Policlinico San Martino - IRCCS, Genoa, Italy. ${ }^{3}$ Microbiology Unit, Azienda Ospedaliera Universitaria Pisana, Pisa, Italy. ${ }^{4}$ Emergency Medicine Department, University of Pisa, Pisa, Italy. Infectious Diseases Division, Department of Medicine, University of Udine and Azienda Sanitaria Universitaria Integrata di Udine, Udine, Italy. ${ }^{6}$ Department of Economics and Finance, University of Rome "Tor Vergata", Rome, Italy

Received: 1 November 2019 Accepted: 14 January 2020

Published online: 30 January 2020

\section{References}

1. European Centre for Disease Prevention and Control. Annual report of the European Antimicrobial Resistance Surveillance Network (EARS-Net) (2018). Available from: https://ecdc.europa.eu/en/publications-data/surveillanceantimicrobial-resistance-europe-2017 Accessed 30 Apr 2019.

2. European Centre for Disease Prevention and Control. Rapid risk assessment: Carbapenem-resistant Enterobacteriaceae - first update 4 June 2018 Stockholm: ECDC (2018). Available from: https://ecdc.europa.eu/sites/portal/ files/documents/RRA-Enterobacteriaceae-Carbapenems-European-Unioncountries.pdf. Accessed 30 Apr 2019.

3. Cassini A, Högberg LD, Plachouras D, Quattrocchi A, Hoxha A, Simonsen GS, et al. Attributable deaths and disability-adjusted life-years caused by infections with antibiotic-resistant bacteria in the EU and the European economic area in 2015: a population-level modelling analysis. Lancet Infect Dis. 2019;19:56-66

4. Ben-David D, Kordevani R, Keller N, Tal I, Marzel A, Gal-Mor O, et al. Outcome of carbapenem resistant Klebsiella pneumoniae bloodstream infections. Clin Microbiol Infect. 2012:18:54-60.

5. Borer A, Saidel-Odes L, Riesenberg K, Eskira S, Peled N, Nativ R, et al. Attributable mortality rate for carbapenem-resistant Klebsiella pneumoniae bacteremia. Infect Control Hosp Epidemiol. 2009;30:972-6.

6. Bassetti M, Giacobbe DR, Giamarellou H, Viscoli C, Daikos GL, Dimopoulos G, et al. Management of KPC-producing Klebsiella pneumoniae infections. Clin Microbiol Infect. 2018:24:133-44.

7. Wang X, Wang Q, Cao B, Sun S, Zhang Y, Gu B, et al. Retrospective observational study from a Chinese network of the impact of combination therapy versus monotherapy on mortality from carbapenem-resistant Enterobacteriaceae bacteremia. Antimicrob Agents Chemother. 2018;63:e01511-8.

8. Tumbarello M, Viale P, Viscoli C, Trecarichi EM, Tumietto F, Marchese A, et al. Predictors of mortality in bloodstream infections caused by Klebsiella pneumoniae carbapenemase-producing K. pneumoniae: importance of combination therapy. Clin Infect Dis. 2012;55:943-50.

9. Falcone M, Russo A, lacovelli A, Restuccia G, Ceccarelli G, Giordano A, et al. Predictors of outcome in ICU patients with septic shock caused by Klebsiella pneumoniae carbapenemase-producing K. pneumoniae. Clin Microbiol Infect. 2016:22:444-50

10. Falcone M, Paterson D. Spotlight on ceftazidime/avibactam: a new option for MDR Gram-negative infections. J Antimicrob Chemother. 2016;71:2713-22.

11. van Duin D, Lok JJ, Earley M, Cober E, Richter SS, Perez F, et al. Colistin versus ceftazidime-avibactam in the treatment of infections due to carbapenem-resistant Enterobacteriaceae. Clin Infect Dis. 2018;66:163-71.

12. Machuca I, Gutiérrez-Gutiérrez B, Gracia-Ahufinger I, Rivera Espinar F, Cano Á, Guzmán-Puche J, et al. Mortality Associated with bacteremia due to colistin-resistant Klebsiella pneumoniae with high-level meropenem resistance: importance of combination therapy without colistin and carbapenems. Antimicrob Agents Chemother. 2017;61.

13. Seymour CW, Gesten F, Prescott HC, et al. Time to treatment and mortality during mandated emergency Care for Sepsis. N Engl J Med. 2017;376:2235-44.

14. Bernhard M, Lichtenstern C, Eckmann C, Weigand MA. The early antibiotic therapy in septic patients--milestone or sticking point? Crit Care. 2014;18:671.

15. Rodriguez-Gómez J, Pérez-Nadales E, Gutiérrez-Gutiérrez B, Machuca I, Martinez-Martinez L, Rivera F, et al. Prognosis of urinary tract infection caused by KPC-producing Klebsiella pneumoniae: the impact of inappropriate empirical treatment. J Inf Secur. 2019;79:245-52.

16. Cienfuegos-Gallet AV, Ocampo de Los Ríos AM, Sierra Viana P, Ramirez Brinez F, Restrepo Castro C, Roncancio Villamil G, et al. Risk factors and survival of patients infected with carbapenem-resistant Klebsiella pneumoniae in a KPC endemic setting: a case-control and cohort study. BMC Infect Dis. 2019;19:830.

17. Patel G, Huprikar S, Factor SH, Jenkins SG, Calfee DP. Outcomes of carbapenem-resistant Klebsiella pneumoniae infection and the impact of antimicrobial and adjunctive therapies. Infect Control Hosp Epidemiol. 2008; 29:1099-106.

18. Satlin MJ, Chen L, Patel G, Gomez-Simmonds A, Weston G, Kim AC, et al. Multicenter clinical and molecular epidemiological analysis of bacteremia due to carbapenem-resistant enterobacteriaceae (CRE) in the CRE Epicenter of the United States. Antimicrob Agents Chemother. 2017;61

19. Vincent JL, Moreno R, Takala J, Willatts S, De Mendonça A, Bruining H, et al. The SOFA (Sepsis-related organ failure assessment) score to describe organ dysfunction/failure. Intensive Care Med. 1996;22:707-10.

20. Knaus WA, Knaus WA, Draper EA, Wagner DP, Zimmerman JE. APACHE II: a severity of disease classification system. Crit Care Med. 1985;13:818-29.

21. CDC/NHSN Surveillance Definitions for Specific Types of Infections. Available at: https://www.cdc.gov/nhsn/PDFs/pscManual/17pscNosInfDef_current.pdf. Accessed: 20 May 2019.

22. Cangemi R, Falcone M, Taliani G, Calvieri C, Tiseo G, Romiti GF, et al. Corticosteroid use and incident myocardial infarction in adults hospitalized for community-acquired pneumonia. Ann Am Thorac Soc. 2019;16:91-8.

23. Friedman ND, Kaye KS, Stout JE, McGarry SA, Trivette SL, Briggs JP, et al. Health care associated bloodstream infections in adults: a reason to change the accepted definition of community-acquired infections. Ann Intern Med. 2002;137:791-7.

24. Singer M, Deutschman CS, Seymour CW, Shankar-Hari M, Annane D, Bauer $M$, et al. The third international consensus definitions for Sepsis and septic shock (Sepsis-3). JAMA. 2016;315:801-10.

25. Mayr VD, Dünser MW, Greil V, Jochberger S, Luckner G, Ulmer H, et al. Causes of death and determinants of outcome in critically ill patients. Crit Care. 2006;10:R154.

26. Coly-Mycin ${ }^{\circledast}$ M Parenteral (Colistimethate for Injection, USP). FDA. Available at: https://wnw.accessdata.fda.gov/drugsatfda_docs/label/2009/050108s026lbl.pdf.

27. Giordano C, Piccoli E, Brucculeri V, Barnini S. A prospective evaluation of two rapid phenotypical antimicrobial susceptibility technologies for the diagnostic stewardship of sepsis. Biomed Res Int. 2018;2018:6976923.

28. European Committee on Antimicrobial Susceptibility Testing (EUCAST). The European Committee on Antimicrobial Susceptibility Testing. Breakpoint tables for interpretation of MICs and zone diameters. Version 9.0, 2019. http://www.eucast.org. Accessed: 30 April 2019.

29. Kumar A, Roberts D, Wood KE, Light B, Parrillo JE, Sharma S, et al. Duration of hypotension before initiation of effective antimicrobial therapy is the critical determinant of survival in human septic shock. Crit Care Med. 2006; 34:1589-96.

30. Weiss SL, Fitzgerald JC, Balamuth F, Alpern ER, Lavelle J, Chilutti M, Grundmeier R. Delayed antimicrobial therapy increases mortality and organ dysfunction duration in pediatric sepsis. Crit Care Med. 2014;2:2409-17.

31. Ferrer R, Martin-Loeches I, Phillips G, Osborn TM, Townsend S, Dellinger RP. Empiric antibiotic treatment reduces mortality in severe sepsis and septic shock from the first hour: results from a guideline-based performance improvement program. Crit Care Med. 2014;42:1749-55.

32. Ferrer $R$, Artigas $A$, Suarez D, Palencia E, Levy MM, Arenzana A, et al. Effectiveness of treatments for severe sepsis: a prospective, multicenter, observational study. Am J Respir Crit Care Med. 2009;180:861-6.

33. Zhang D, Micek ST, Kollef MH. Time to appropriate antibiotic therapy is an independent determinant of post-infection ICU and hospital lengths of stay in patients with sepsis. Crit Care Med. 2015;43:2133-40. 
34. Tabak YP, Vankeepuram L, Ye G, Jeffers K, Gupta V, Murray PR. Blood culture turnaround time in U.S. acute care hospitals and implications for laboratory process optimization. J Clin Microbiol. 2018;56:e00500-18.

35. Cano A, Gutiérrez-Gutiérrez B, Machuca I, Gracia-Ahufinger I, Pérez-Nadales E, Causse M, et al. Risks of infection and mortality among patients colonized with Klebsiella pneumoniae carbapenemase-producing K. pneumoniae: validation of scores and proposal for management. Clin Infect Dis. 2018;66 1204-10.

36. Lima-Morales D, Ávila H, Soldi T, Dalmolin TV, Lutz L, Aquino V, et al. Rapid detection of carbapenemase production directly from blood culture by colorimetric methods: evaluation in a routine microbiology laboratory. J Clin Microbiol. 2018;56:e00325-18.

37. Dortet L, Agathine A, Naas T, Cuzon G, Poirel L, Nordmann P. Evaluation of the RAPIDEC ${ }^{\bullet}$ CARBA NP, the rapid CARB screen ${ }^{\bullet}$ and the carba NP test for biochemical detection of carbapenemase-producing Enterobacteriaceae. J Antimicrob Chemother. 2015;70:3014-22.

38. Cisneros JM, Rosso-Fernández CM, Roca-Oporto C, De Pascale G, JiménezJorge S, Fernández-Hinojosa E, et al. Colistin versus meropenem in the empirical treatment of ventilator-associated pneumonia (magic bullet study): an investigator-driven, open-label, randomized, noninferiority controlled trial. Crit Care. 2019;23:383. https://doi.org/10.1186/s13054-019-2627-y.

39. Motsch J, Murta de Oliveira C, Stus V, Köksal I, Lyulko O, Boucher HW, et al. RESTORE-IMI 1: a multicenter, randomized, double-blind trial comparing efficacy and safety of imipenem/relebactam vs colistin plus imipenem in patients with imipenem-nonsusceptible bacterial infections. Clin Infect Dis. 2019. [Epub ahead of print]

40. Kwon KH, Oh JY, Yoon YS, Jeong YJ, Kim KS, Shin SJ, et al. Colistin treatment in carbapenem-resistant Acinetobacter baumannii pneumonia patients: incidence of nephrotoxicity and outcomes. Int J Antimicrob Agents. 2015; 45:605-9.

41. Chusri S, Singkhamanan K, Wanitsuwan W, Suphasynth Y, Kositpantawong $\mathrm{N}$, Panthuwong S, et al. Adjunctive therapy of intravenous colistin to intravenous tigecycline for adult patients with non-bacteremic post-surgical intra-abdominal infection due to carbapenem-resistant Acinetobacter baumannii. J Infect Chemother. 2019;25:681-6.

42. Mimoz O, Petitpas F, Grégoire N, Gobin P, Marchand S, Couet W. Colistin distribution in the peritoneal fluid of a patient with severe peritonitis. Antimicrob Agents Chemother. 2012;56:4035-6.

43. Fantin B, Poujade J, Grégoire N, Chau F, Roujansky A, Kieffer N, et al. The inoculum effect of Escherichia coli expressing mcr-1 or not on colistin activity in a murine model of peritonitis. Clin Microbiol Infect. 2019;25:1563.e5-8.

44. Luque S, Escaño C, Sorli L, Li J, Campillo N, Horcajada JP, et al. Urinary concentrations of colistimethate and formed colistin after intravenous administration in patients with multidrug-resistant Gram-negative bacterial infections. Antimicrob Agents Chemother. 2017;61

45. Matuschek E, Åhman J, Webster C, Kahlmeter G. Antimicrobial susceptibility testing of colistin - evaluation of seven commercial MIC products against standard broth microdilution for Escherichia coli, Klebsiella pneumoniae, Pseudomonas aeruginosa, and Acinetobacter spp. Clin Microbiol Infect. 2018; 24:865-70

\section{Publisher's Note}

Springer Nature remains neutral with regard to jurisdictional claims in published maps and institutional affiliations.

Ready to submit your research? Choose BMC and benefit from:

- fast, convenient online submission

- thorough peer review by experienced researchers in your field

- rapid publication on acceptance

- support for research data, including large and complex data types

- gold Open Access which fosters wider collaboration and increased citations

- maximum visibility for your research: over $100 \mathrm{M}$ website views per year

At $\mathrm{BMC}$, research is always in progress.

Learn more biomedcentral.com/submissions 